\section{A FORTRAN 77 program for the runs test}

\author{
EDWARD F. KRIEG, JR. \\ Tulane University, New Orleans, Louisiana
}

When linear or nonlinear regression analysis is done, it is often useful, if not necessary, to examine the residuals in order to determine whether or not the model is appropriate or whether outliers are present. Swed and Eisenhart (1943) developed a statistic that can be used to test the randomness of a sequence of residuals. The signs of the residuals ( + or - ) are lined up in the order in which they appear when going from left to right along the $x$ axis. The number of positive and negative residuals, as well as the number of distinct groupings of pluses and minuses, are then counted. For example, in the sequence of residuals ++-+-+++-- , there are 6 pluses, 4 minuses, and 6 distinct groupings or "runs." Usually a lower-tailed test of significance has been employed by calculating the probability of getting $U$ or fewer runs in a random arrangement of $m$ pluses and $n$ minuses. When the calculated probability is less than .05 , the null hypothesis that the order of the residuals is random is rejected. This indicates that the model being used is inappropriate, or it may indicate the presence of outliers.

The program provides exact and normal approximations for lower-, upper-, and two-tailed tests of significance. The reason for including the upper-tailed probability is that if there is a significantly greater number of runs than would occur by chance alone, this might also indicate an inappropriate model.

Methodology. The exact probabilities for the runs test are calculated using formulas provided by Bennett and Franklin (1954). The two-tailed probability is calculated in the same manner as the two-tailed probability of the

\footnotetext{
Address correspondence to the author at the National Institute for Occupational Safety and Health, 4676 Columbia Pkwy. (C-22), Cincinnati, $\mathrm{OH} 45226$.
}

Fisher exact test. All the probabilities in the distribution that are less than or equal to the probability of getting $U$ runs are summed. The mean and variance of the runs distributions, as well as the normal approximations of the probabilities, are calculated as described by Draper and Smith (1966). A correction for continuity is used in calculating the standard normal deviate by bringing the number of runs a half-step closer to the mean of the distribution by either adding $1 / 2$ to or subtracting $1 / 2$ from the number of runs.

Input and Output. One enters the number of positive residuals, the number of negative residuals, and the number of runs. The output includes the exact and normal approximations of the runs probabilities, and the runs distribution parameters. Output can be read from a display screen, and it can also be directed to the default printer of the user's system.

Language and Computer. The program was written in FORTRAN 77 and was compiled on an IBM PC/AT using the IBM Professional FORTRAN Compiler. All calculations are done in double precision. To run this program, it is necessary to have an IBM PC (DOS 2.1 or higher) or compatible with $64 \mathrm{~K}$ of memory for program execution as well as a math coprocessor compatible with the user's system.

Availability. Copies of the source code and the compiled code may be obtained from the author. Please include a floppy diskette.

\section{REFERENCES}

Bennett, C. A., Frankun, N. L. (1954). Statistical analysis in chemistry and the chemical industry. New York: Wiley.

DRAPER, N. R., \& SMITH, H. (1966). Applied regression analysis. New York: Wiley.

SWED, F. S., \& EISENHART, C. (1943). Tables for testing randomness of grouping in a sequence of alternatives. Annals of Mathematical Statistics, 14, 66-87.

(Revision accepted for publication February 2, 1988.) 